https://helda.helsinki.fi

\title{
Oral surgery in liver transplant candidates : a retrospective study on delayed bleeding and other complications
}

\section{Helenius-Hietala, Jaana}

2016-05

Helenius-Hietala , J , Åberg , F , Meurman , J H , Nordin , A \& Isoniemi , H 2016 , ' Oral surgery in liver transplant candidates : a retrospective study on delayed bleeding and other complications ' , Oral surgery, oral medicine, oral pathology and oral radiology, vol. 121, no. 5 , pp. 490-495 . https://doi.org/10.1016/j.0000.2016.01.025

http://hdl.handle.net/10138/223984

https://doi.org/10.1016/j.0000.2016.01.025

publishedVersion

Downloaded from Helda, University of Helsinki institutional repository.

This is an electronic reprint of the original article.

This reprint may differ from the original in pagination and typographic detail.

Please cite the original version. 


\title{
Oral surgery in liver transplant candidates: a retrospective study on delayed bleeding and other complications
}

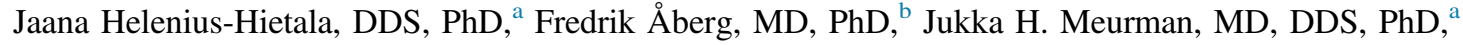 \\ Arno Nordin, $\mathrm{MD}, \mathrm{PhD},{ }^{\mathrm{c}}$ and Helena Isoniemi, $\mathrm{MD}, \mathrm{PhD}^{\mathrm{c}}$
}

Objective. Untreated dental infections pose a threat for immunocompromised liver transplant (LT) recipients. Therefore, pretransplant dental evaluations are recommended. However, risk of bleeding should be considered among patients with endstage liver disease, and prophylactic blood transfusions may be used to prevent bleeding. We performed a retrospective study of the incidence of and risk factors for oral surgery-related bleeding in candidates for LT and hypothesized that complications may occur despite preoperative and perioperative hemostatic actions.

Study Design. One hundred thirty-four patients who had tooth extractions performed by oral and maxillofacial surgeons before LT were studied. The primary endpoint was bleeding between 24 hours and 2 weeks after extraction. Bleeding risk was analyzed by preoperative platelet (PLT) count and international normalized ratio (INR). Invasiveness of procedures, severity of liver disease, PLT, INR, prophylactic transfusions of PLT, fresh frozen plasma, and tranexamic acid (TA) were included in univariate and multivariate logistic regression analyses to further assess risk.

Results. Twelve patients exhibited minor bleeding; four despite PLT $>100 \times 10^{9} / \mathrm{L}$ and INR $<1.5$. Increased bleeding associated with INR and prophylactic transfusions by univariate analysis; by multivariate analyses, prophylactic TA (odds ratio $[\mathrm{OR}]=8.0 ; 95 \%$ confidence interval $[\mathrm{Cl}]$ 1.7-37.0), and PLT $(\mathrm{OR}=8.3 ; 95 \% \mathrm{Cl} 1.1-62.7)$ remained significant.

Conclusions. Most extractions were safe, but prophylactic transfusions did not ensure adequate hemostasis. Local hemostatic measures and close follow-up are warranted. (Oral Surg Oral Med Oral Pathol Oral Radiol 2016;121:490-495)

Pretransplant dental treatment is a prerequisite in many liver transplantation (LT) programs. This requirement aims to prevent posttransplant infectious complications, such as bacteremia and sepsis, which could be fatal in immunocompromised patients. ${ }^{1,2}$ In particular, patients with chronic liver disease (CLD) have shown poor oral health with a high prevalence of dental infections in pre-LT dental evaluations. ${ }^{3}$ Often, teeth with obvious severe infections are extracted after administering antibiotic prophylaxis. Although there is no scientific evidence in the literature to support antibiotic prophylaxis for this patient group, our clinic has adopted this protocol to prevent serious postoperative infectious complications in these high-risk patients. Patients with CLD also run a high risk of bleeding after oral surgery because of CLD-associated coagulopathy. ${ }^{4}$ Reported incidences of bleeding after oral surgery in An abstract from this research was presented at the Scandinavian Transplantation Society meeting in Copenhagen (May 2014), PER IADR in Dubrovnik (September 2014), and at AAOM meeting in San Diego (April 2015).

Funding for this study was provided by the Helsinki University Hospital funds (EVOT1020Y0011).

${ }^{\mathrm{a}}$ Department of Oral and Maxillofacial Diseases, University of Helsinki and Helsinki University Hospital, Helsinki, Finland.

${ }^{\mathrm{b}}$ Department of Gastroenterology, Helsinki University Hospital, Helsinki, Finland.

${ }^{c}$ Transplantation and Liver Surgery Clinic, Helsinki University Hospital, Helsinki, Finland.

Received for publication Aug 25, 2015; returned for revision Jan 18, 2016; accepted for publication Jan 24, 2016.

(c) 2016 Elsevier Inc. All rights reserved.

2212-4403/\$ - see front matter

http://dx.doi.org/10.1016/j.0ooo.2016.01.025 patients with CLD vary from $0 \%$ to $56 \% .^{4-8}$ However, previous studies were limited by small cohorts (9-90 patients), and those patients often had wellcompensated CLD. ${ }^{6}$ Earlier studies that specifically studied LT candidates, that is, patients with advanced or end-stage liver disease, included only 23 to 36 patients. $^{4,5,7,8}$

Platelets (PLT), fresh frozen plasma (FFP), and tranexamic acid (TA) are typically administered before oral surgery in an attempt to prevent bleeding despite the lack of firm evidence to support this strategy. It has been suggested that the risk of bleeding might be predicted by an elevated international normalized ratio (INR) or thrombocytopenia; therefore, prophylactic transfusions of FFP and PLT have been justified to prevent bleeding complications. Because of the high fibrinolytic activity in the oral cavity, systemic antifibrinolytics, such as TA, are sometimes used in addition to the local antifibrinolytics. However, the systemic

\section{Statement of Clinical Relevance}

International normalized ratio and platelet count do not reliably predict bleeding after tooth extractions in liver transplant candidates because of rebalanced hemostasis. Hence, routine prophylactic replacement therapy may be ineffective. Alternatively, local hemostatic measures and close postoperative follow-up by a skilled clinician are recommended. 
approach remains controversial because of the risk of thrombotic events. Nevertheless, the effect of these blood products lasts for only a few hours; consequently, they may not have an impact on delayed bleeding that can occur several days later.

The primary aim of the current retrospective study was to investigate the incidence and risk factors of bleeding complications following tooth extractions in candidates for LT. We also investigated the effects of preprocedural transfusions to prevent post-oral surgery bleeding. A secondary aim was to investigate other complications related to oral surgery. We hypothesized that complications might occur, despite preoperative and perioperative hemostatic actions.

\section{MATERIALS AND METHODS}

This retrospective study was conducted in agreement with the Declaration of Helsinki and was received exemption from institutional review board approval. The primary endpoint of the study was oral bleeding related to oral surgery that appeared within 2 weeks from surgery. Replacement therapy was considered effective when good hemostasis was achieved during the procedure and the patient did not show any delayed bleeding.

During 2000-2006, a total of 306 adult LTs were performed in Finland. Of these patients, 263 had dental evaluations before LT at the Department of Oral and Maxillofacial Diseases, Helsinki University Hospital, Helsinki, Finland. As part of routine practice before LT the Transplantation and Liver Surgery Clinic referred patients to an oral and maxillofacial (OMF) surgeon in our department for dental treatments.

We retrieved data from patient medical records, tomographic radiographs, and the hospital laboratory database. Medical records were reviewed when any oral surgery-related bleeding complication required hospital care. These events were considered bleeding complications in the present study. We defined "bleeding" as continuous or delayed bleeding, observed at least 24 hours following tooth extractions, which required a return visit to an OMF surgeon for treatment. The bleeding was recorded by either a medical doctor or nurse at the hospital ward. The amount of blood loss was not recorded.

Data collected from the patient records included type of liver disease, number of teeth, number of extracted teeth, number of difficult extractions (impacted wisdom teeth, representing invasive procedures), oral surgery related complications, and preoperative PLT and INR values, as well as intravenous (IV) preprocedural replacement therapies: PLT (8-16 IU), FFP (2-4 IU), and TA (1-3 g oral or IV administration). These replacement therapies were given prophylactically, based on the anesthesiologist's individual clinical assessment of bleeding risk, when the PLT count was low and the INR was high.

The Model for End-stage Liver Disease (MELD) was used to categorize patients in this study. MELD scores reflect the severity of liver disease, and they are used to predict the risk of death. The scores were calculated with the Mayo Clinic online calculator. The calculation included blood creatinine, bilirubin, and INR values recorded at the time the patient was listed for LT. The MELD equation assumed an upper limit of $350 \mu \mathrm{M}$ for plasma creatinine.

\section{Statistical analysis}

Data were analyzed with PASW statistical software, version 17.0 (SPSS Inc., Chicago, IL). The $\chi^{2}$ test was used to evaluate categorical variables, and the Mann-Whitney test was used for continuous variables. The effect of various factors on bleeding risk was assessed by univariate and multivariate logistic regression analyses. A two-sided $P<.05$ was considered significant.

\section{RESULTS}

Patient demographic characteristics are given in Table I. The final study population comprised 134 patients who required tooth extractions before LT. Most patients returned to the in-patient clinic for at least one night following tooth extractions. In some cases, patients stayed overnight at the Department of Oral and Maxillofacial Diseases. In either case, postoperative follow-up was readily available. TA was routinely used locally. It was applied in liquid form to saturate gauzes, which were then placed tightly over alveolar sockets after tooth extractions. Other local hemostatic measures were provided to all patients, including careful, tight suturing of the wounds and close follow-up in the recovery room.

One patient had acute liver failure, and all others had CLD. Of the patients, 12 exhibited bleeding complications more than 24 hours postoperatively, which required treatment from an OMF surgeon. In the present study, all bleeding complications were minor and fairly simple to treat. In most cases, bleeding occurred 1 to 2 days postoperatively, but one case exhibited excessive bleeding 8 days postoperatively. That patient had remained in the ward; thus, treatment was easily available. In cases of delayed bleeding, an OMF surgeon was called, and the alveolar socket was either resutured or local hemostatic agents were administered (e.g., with local TA surges/rinse or with gelatin sponges), and tight gauze pads were placed on bleeding alveolar sockets.

The average number of teeth extracted was five (range 1-20); four patients required full mouth 
Table I. Basic characteristics of the study population

\begin{tabular}{|c|c|c|c|c|}
\hline & $\begin{array}{c}\text { All } \\
\text { patients; } \\
n(\%)\end{array}$ & $\begin{array}{c}\text { Patients with } \\
\text { bleeding; } \\
n(\%)\end{array}$ & $\begin{array}{c}\text { Patients } \\
\text { without } \\
\text { bleeding; } \\
n(\%)\end{array}$ & $P$ \\
\hline Patients & 134 & 12 & 122 & \\
\hline Age & $50(11)$ & $45(12)$ & $51(11)$ & .088 \\
\hline Gender, male & $54(40)$ & $6(50)$ & $48(39)$ & .47 \\
\hline Etiology of liver disease & & & & .017 \\
\hline \multicolumn{5}{|l|}{ Chronic liver disease } \\
\hline $\mathrm{PBC}$ & $20(15)$ & $3(25)$ & $17(14)$ & \\
\hline PSC & $33(25)$ & $1(8)$ & $32(26)$ & \\
\hline Alcohol & $27(20)$ & $4(33)$ & $23(19)$ & \\
\hline $\begin{array}{l}\text { Cryptogenic } \\
\text { cirrhosis }\end{array}$ & $9(7)$ & $0(0)$ & $9(7)$ & \\
\hline Other cirrhosis ${ }^{*}$ & $23(17)$ & $3(25)$ & $20(16)$ & \\
\hline Tumor & $14(10)$ & $0(0)$ & $14(12)$ & \\
\hline Other $^{\dagger}$ & $7(5)$ & $0(0)$ & $7(6)$ & \\
\hline Acute liver failure & $1(1)$ & $1(8)$ & $0(0)$ & \\
\hline $\begin{array}{l}\text { Platelet level }\left(10^{9} / \mathrm{L}\right) \\
\text { mean } \pm \mathrm{SD}\end{array}$ & $134(88)$ & $114(74)$ & $136(90)$ & .48 \\
\hline$<100$ & $66(49)$ & $6(50)$ & $60(49)$ & .96 \\
\hline$<50$ & $13(10)$ & $3(25)$ & $10(8)$ & .06 \\
\hline INR, mean (SD) & $1.5(0.6)$ & $2.0(1.2)$ & $1.4(0.5)$ & .08 \\
\hline$>1.5$ & $47(35)$ & $6(50)$ & $41(34)$ & .26 \\
\hline$>2.0$ & $17(13)$ & $4(33)$ & $13(11)$ & .024 \\
\hline MELD, mean (SD) & $14.4(8.2)$ & $18.8(9.8)$ & $14.0(7.9)$ & .12 \\
\hline $\begin{array}{l}\text { Number of teeth, } \\
\text { mean (SD) }\end{array}$ & $25(6)$ & $26(3)$ & $25(6)$ & .94 \\
\hline $\begin{array}{l}\text { Number of extractions, } \\
\text { mean (SD) }\end{array}$ & $5(4)$ & $5(3)$ & $5(4)$ & .79 \\
\hline$\geq 6$ & $46(34)$ & $4(33)$ & $42(34)$ & \\
\hline $\begin{array}{l}\text { Number of difficult } \\
\text { extractions }\end{array}$ & $26(19)$ & $2(17)$ & $24(20)$ & \\
\hline \multicolumn{5}{|c|}{ Preprocedural hemostatic agents } \\
\hline PLT & $22(16)$ & $6(50)$ & $16(13)$ & .001 \\
\hline FFP & $28(21)$ & $6(50)$ & $22(18)$ & .009 \\
\hline Tranexamic acid & $23(17)$ & $7(58)$ & $16(13)$ & $<.001$ \\
\hline
\end{tabular}

$P B C$, Primary biliary cirrhosis; $P S C$, primary sclerosing cholangitis; $S D$, standard deviation; $I N R$, international normalized ratio; $M E L D$, Model for End-stage Liver Disease; PLT, platelets; FFP, fresh frozen plasma.

Bold values represent statistical significance.

*Other cirrhosis patients include those with autoimmune cirrhosis or viral hepatitis.

${ }^{\dagger}$ Other patients include patients with biliary atresia or metabolic liver disease.

extractions. The number of teeth extracted or the difficulty of extractions did not correlate with bleeding episodes; three patients had bleeding even after simple extractions of one or two teeth. However, another three patients experienced bleeding after sinus perforations as a result of extraction of molars in the upper jaw.

Compared with patients with no bleeding, patients with bleeding tended to have higher mean MELD scores (19 vs 14 ), higher INRs (2.0 vs 1.4 ), and lower PLT counts $\left(114\right.$ vs $\left.136 \times 10^{9} / \mathrm{L}\right)$. Although these differences were not significant, a significantly higher proportion of patients with bleeding had INR $>2.0$, compared with those without bleeding (Table I). The
Table II. Bleeding incidences according to different INR or platelet levels

\begin{tabular}{|c|c|c|c|}
\hline & $I N R<1.5 ; n(\%)$ & $I N R>1.5 ; n(\%)$ & $P$ \\
\hline \multicolumn{4}{|c|}{ Platelet level $\left(10^{9} / \mathrm{L}\right)$} \\
\hline$>100$ & $4 / 54(7)$ & 2/14 (14) & .45 \\
\hline$<100$ & $2 / 33(6)$ & $4 / 33(12)$ & .39 \\
\hline$P$ & .81 & .84 & \\
\hline$>50$ & $6 / 44(14)$ & $3 / 37(8)$ & .99 \\
\hline$<50$ & $0 / 4(0)$ & $3 / 7(43)$ & .067 \\
\hline$P$ & .37 & .01 & \\
\hline
\end{tabular}

$I N R$, International normalized ratio.

Bold values represent statistical significance.

etiology of liver disease significantly affected bleeding risk. Among the patients with primary biliary cirrhosis, alcohol cirrhosis, and other cirrhosis (those with autoimmune cirrhosis or viral hepatitis), a substantially higher proportion exhibited bleeding compared with groups with other etiologies, such as primary sclerosing cholangitis patients (11 vs 1, respectively).

Among all patients, prophylactic transfusions were administered as follows: $16 \%$ received PLT (mean 8 IU), $21 \%$ received FFP (mean $4 \mathrm{IU}$ ), and $17 \%$ received TA (mean $1 \mathrm{~g} \times 3$ ). In addition, one patient received prophylactic 1250 IU Fibrogrammin IV (purified concentrate of blood coagulation factor XIII; CSL Behring, UK) and $2 \mathrm{~g}$ fibrinogen (glycoprotein, factor I, which is converted into fibrin). Preprocedural transfusions were performed significantly more often in patients with bleeding than in patients without bleeding (Table I). In general, prophylactic FFP and PLT transfusions were administered when INR exceeded the threshold of 2.0 and PLT counts fell under $100 \times 10^{9} / \mathrm{L}$. However, marked individual variations in these thresholds existed; therefore, these thresholds were relaxed on the basis of other contributing factors, including the type of operation, patient blood volume status, the degree of portal hypertension, a history of bleeding tendency, and the perceived bleeding risk.

When stratified by INR and PLT levels, the highest bleeding incidences occurred among patients with simultaneous INR $>1.5$ and PLT counts $<50 \times 10^{9} / \mathrm{L}$ (Table II). Nonetheless, four patients exhibited bleeding, despite PLT counts $>100 \times 10^{9} / \mathrm{L}$ and INRs $<1.5$. Regardless of the pre-oral surgery PLT level prophylactic PLT transfusions were associated with an increased risk of bleeding (Table III). A similar association was found between INR and prophylactic FFP transfusions, although this association was significant only among patients with INRs $>1.5$ (Table III).

In univariate logistic regression analyses, INR and prophylactic transfusion of hemostatic agents were associated with higher bleeding risk (Table IV). In the 
Table III. Effect of preprocedural transfusion of platelets or FFP on bleeding incidences according to preprocedural platelet $\left(10^{9} / \mathrm{L}\right)$ or INR level

\begin{tabular}{lcc}
\hline & Bleeding incidence; $n(\%)$ & $P$ \\
\hline PLT $<100$ & $5 / 21(24)$ & $\mathbf{. 0 0 4}$ \\
PLT transfusion & $1 / 45(2)$ & \\
No PLT transfusion & & $\mathbf{. 0 0 1}$ \\
PLT $>100$ & $1 / 1(100)$ & \\
$\quad$ PLT transfusion & $5 / 66(8)$ & $\mathbf{. 0 4 8}$ \\
$\quad$ No PLT transfusion & $5 / 22(23)$ & \\
INR $>1.5$ & $1 / 25(4)$ & .34 \\
FFP transfusion & & \\
No FFP transfusion & $1 / 6(17)$ & \\
INR $<1.5$ & $5 / 79(6)$ & \\
FFP transfusion & & \\
No FFP transfusion &
\end{tabular}

FFP, Fresh frozen plasma; INR, international normalized ratio; PLT, platelets (shown in $10^{9} / \mathrm{L}$ ).

Bold values represent statistical significance.

multivariate analyses, prophylactic TA use (and PLT transfusion) remained significant (Table IV).

Other complications after oral surgery were rare. Four patients had high fever postoperatively, and they were treated with IV antibiotic therapy. Milder complications included one case with slight swelling in the facial region. In another instance, the medical status of a patient with cirrhosis worsened considerably after extraction of seven teeth, and the patient needed hospital treatment.

\section{DISCUSSION}

We aimed to investigate the incidence of bleeding complications from oral surgery among candidates for LT. We also wanted to investigate how preprocedural replacement therapy affected bleeding and whether the laboratory values typically used to indicate bleeding risk could predict bleeding risk in this group of patients.

This study is one of the largest, to date, on bleeding after oral surgery in patients with end-stage liver disease. We found that the majority of tooth extractions (91\%) were performed safely, without bleeding complications. The highest bleeding incidences were observed among patients with concurrent, severe thrombocytopenia (PLT $<50 \times 10^{9} / \mathrm{L}$ ) and impaired coagulation factor synthesis (INR $>1.5$ ). Preprocedural blood-product replacement therapy with PLT, FFP, or TA failed to ensure hemostasis. These results implied that prophylactic replacement therapies were ineffective. However, it remained unclear whether this ineffectiveness was secondary to the types of agents, the doses, or the timing employed.

The bleeding frequency of $9 \%$ found in our study was within the range of $3 \%$ to $17 \%$, as reported in previous studies that included only LT candidates. $4,5,7,8$ Earlier studies, however, were markedly different from our study in terms of the severity or type of liver disease and the size of the patient population (the previous studies included less than 40 patients). For example, in a recent study by Perdigao et al., ${ }^{5}$ only one bleeding event occurred among 23 patients, and local hemostatic measures (TA and absorbable sponges) were sufficient to correct that postoperative bleeding complication, without the need for blood transfusions.

Recent studies have challenged the long-held belief that patients with liver disease are at increased risk of bleeding because of impaired coagulation factor synthesis and mild to moderate thrombocytopenia and that this bleeding risk can be reduced with prophylactic FFP or PLT transfusions delivered before invasive procedures. ${ }^{9,10}$ Growing evidence has shown that even in end-stage liver disease, concomitant with deficiencies in natural anticoagulants the body can produce elevated levels of factor VIII and von Willebrand factor to rebalance the hemostatic state and thus preserve the capacity to generate thrombin and fibrin. ${ }^{9-12}$ INR and platelet counts do not reflect this rebalanced hemostasis, and therefore, they are poor predictors of bleeding in liver disease. ${ }^{9,10}$ Moreover, it has been shown that prophylactic blood product transfusions are often ineffective $^{13,14}$ and may even be harmful ${ }^{15-17}$ because the associated fluid overload may increase bleeding risk by aggravating portal hypertension. ${ }^{18,19}$ Consequently, recent recommendations regarding invasive procedures in patients with end-stage liver disease have emphasized the need to monitor patients closely when bleeding occurs, and they strongly discourage the routine use of prophylactic transfusions. ${ }^{10,20,21}$ Our present findings are in full agreement with those recommendations. Perdigao et al. $^{5}$ found a low postoperative bleeding incidence without prophylactic blood transfusions among patients with advanced cirrhosis, and a study by Stanca et al. ${ }^{7}$ showed that intranasal desmopressin was as effective as a blood transfusion for achieving hemostasis. Moreover, the effects of blood product replacement therapy are transient; thus, this strategy may not prevent delayed bleeding.

Hyperfibrinolysis may occur in advanced liver disease. This condition is potentially a common cause of delayed bleeding after various procedures. ${ }^{10}$ There are no readily available methods for detecting hyperfibrinolysis in routine practice. It is known, however, that the oral cavity has high fibrinolytic activity, ${ }^{22}$ which supports the use of antifibrinolytics, such as TA, after tooth extraction, particularly in patients with liver disease. Nevertheless, in our analysis, systemic TA failed to protect from delayed bleeding. However, because the effect of TA lasts only for some hours, it might be speculated that extended TA use over several days could protect from delayed bleeding more effectively. 
Table IV. Factors associated with bleeding complications by univariate and multivariate logistic regression analyses

\begin{tabular}{|c|c|c|c|c|c|c|}
\hline & \multicolumn{2}{|c|}{ Univariate } & \multicolumn{2}{|c|}{ Multivariate model $1^{*}$} & \multicolumn{2}{|c|}{ Multivariate model $2^{\dagger}$} \\
\hline & OR $(95 \% C I)$ & $P$ & OR $(95 \% C I)$ & $P$ & OR $(95 \% C I)$ & $P$ \\
\hline Number of tooth extractions & $0.99(0.86-1.15)$ & .92 & & & $0.93(0.78-1.12)$ & .44 \\
\hline Difficult extraction (e.g., impaction) & $0.82(0.17-3.98)$ & .80 & & & $0.82(0.12-5.54)$ & .84 \\
\hline Platelet level & $1.00(0.99-1.01)$ & .42 & & & $1.00(0.99-1.01)$ & .58 \\
\hline INR & $3.05(1.31-7.10)$ & .01 & $1.58(0.43-5.80)$ & .49 & $1.30(0.31-5.47)$ & .72 \\
\hline MELD score & $1.07(1.00-1.14)$ & .06 & $1.01(0.90-1.13)$ & .89 & $1.01(0.91-1.13)$ & .83 \\
\hline Preprocedural platelet transfusion & $6.63(1.90-23.0)$ & .003 & $5.45(1.0-29.8)$ & .051 & $8.32(1.10-62.7)$ & .04 \\
\hline Preprocedural FFP transfusion & $4.55(1.34-15.4)$ & .02 & $0.62(0.09-4.24)$ & .62 & $0.66(0.09-4.97)$ & 69 \\
\hline Preprocedural tranexamic acid use & $9.28(2.63-32.8)$ & .001 & $6.65(1.58-28.0)$ & .01 & $7.99(1.72-37.0)$ & .008 \\
\hline
\end{tabular}

$O R$, Odds ratio; $C I$, confidence interval; $I N R$, international normalized ratio; $M E L D$, Model for End-stage Liver Disease; $F F P$, fresh frozen plasma. Bold values represent statistical significance.

*Only factors with $P<.1$ on univariate analysis included.

${ }^{\dagger}$ All univariate factors included.

Our study had several limitations. First, it was retrospective in nature. Also, although bleeding risk assessments in clinical practice have largely been based on INR and PLT counts, we did not account for other potential factors that may have determined a need for preprocedural transfusions. Nevertheless, our results partly confirmed the study hypothesis that complications may occur despite preoperative and perioperative hemostatic measures.

Douglas et al. ${ }^{23}$ recommended that for blood product replacement therapy in oral surgery, FFP should be administered to patients with INRs $\geq 3$.0. In contrast, the results of the present study suggested that the need for prophylactic FFP transfusions should be carefully evaluated. Our results were consistent with several previous studies showing that INR was indeed a poor predictor of the risk for bleeding in patients with end-stage liver disease. ${ }^{9,10}$ Individual evaluations are required to weigh the benefits and drawbacks of transfusing PLT or other blood clotting factors in patients with cirrhosis.

\section{CONCLUSIONS}

Our results showed that the risk of bleeding is difficult to predict in patients with cirrhosis but that frequency of delayed bleeding is in general low. We recommend that atraumatic surgical techniques be applied by qualified practitioners along with the use of local hemostatic agents and close postoperative follow-up. Finally, our results suggest that use of prophylactic blood coagulation factors requires individual assessment.

\section{REFERENCES}

1. Guggenheimer J, Eghtesad B, Stock DJ. Dental management of the (solid) organ transplant patient. Oral Surg Oral Med Oral Pathol Oral Radiol Endod. 2003;95:383-389.

2. Helenius-Hietala J, Åberg F, Meurman JH, Isoniemi H. Increased infection risk post-liver transplant without pre-transplant dental treatment. Oral Dis. 2013;19:271-278.
3. Helenius-Hietala J, Meurman JH, Höckerstedt K, Lindqvist C, Isoniemi $\mathrm{H}$. Effect of the aetiology and severity of liver disease on oral health and dental treatment prior to transplantation. Transpl Int. 2012;25:158-165.

4. Ward BB, Weideman EM. Long-term postoperative bleeding after dentoalveolar surgery in the pretransplant liver failure patient. J Oral Maxillofac Surg. 2006;64:1469-1474.

5. Perdigao JP, de Almeida PC, Rocha TD, et al. Postoperative bleeding after dental extraction in liver pretransplant patients. J Oral Maxillofac Surg. 2012;70:177-184.

6. Hong CH, Scobey MW, Napenas JJ, Brennan MT, Lockhart PB. Dental postoperative bleeding complications in patients with suspected and documented liver disease. Oral Dis. 2012;18: 661-666.

7. Stanca CM, Montazem AH, Lawal A, Zhang JX, Schiano TD. Intranasal desmopressin versus blood transfusion in cirrhotic patients with coagulopathy undergoing dental extraction: a randomized controlled trial. J Oral Maxillofac Surg. 2010;68: 138-143.

8. Valerin MA, Napeñas JJ, Brennan MT, Fox PC, Lockhart PB. Modified Child-Pugh score as a marker for postoperative bleeding from invasive dental procedures. Oral Surg Oral Med Oral Pathol Oral Radiol Endod. 2007;104:56.

9. Tripodi Armando MP. The coagulopathy of chronic liver disease. N Engl J Med. 2011;365:147-156.

10. Lisman T, Caldwell SH, Burroughs AK, et al. Hemostasis and thrombosis in patients with liver disease: the ups and downs. J Hepatol. 2010;53:362-371.

11. Tripodi A, Salerno F, Chantarangkul V, et al. Evidence of normal thrombin generation in cirrhosis despite abnormal conventional coagulation tests. Hepatology. 2005;41:553-558.

12. Lisman T, Bongers TN, Adelmeijer J, et al. Elevated levels of von Willebrand factor in cirrhosis support platelet adhesion despite reduced functional capacity. Hepatology. 2006;44:53-61.

13. Segal JB, Dzik WH, Clinical TM. Paucity of studies to support that abnormal coagulation test results predict bleeding in the setting of invasive procedures: an evidence-based review. Transfusion. 2005;45:1413-1425.

14. Tripodi A, Chantarangkul V, Primignani M, et al. Thrombin generation in plasma from patients with cirrhosis supplemented with normal plasma: considerations on the efficacy of treatment with fresh-frozen plasma. Intern Emerg Med. 2012;7:139-144.

15. Bhangui P, Laurent A, Amathieu R, Azoulay D. Assessment of risk for non-hepatic surgery in cirrhotic patients. $J$ Hepatol. 2012;57:874-884.

16. de Boer MT, Christensen MC, Asmussen M, et al. The impact of intraoperative transfusion of platelets and red blood cells 
on survival after liver transplantation. Anesth Analg. 2008;106: 32-44.

17. Villanueva C, Colomo A, Bosch A, et al. Transfusion strategies for acute upper gastrointestinal bleeding. $N$ Engl J Med. 2013;368:11-21.

18. Zimmon DS, Kessler RE. Portal pressure-blood volume relationship in cirrhosis. Gut. 1974;15:99-101.

19. Westerkamp AC, Lisman T, Porte RJ. How to minimize blood loss during liver surgery in patients with cirrhosis. $H P B$ (Oxford). 2009;11:453-458.

20. Northup PG, Caldwell SH. Coagulation in liver disease: a guide for the clinician. Clin Gastroenterol Hepatol. 2013;11:10641074.

21. Shah NL, Intagliata NM, Northup PG, Argo CK, Caldwell SH. Procoagulant therapeutics in liver disease: a critique and clinical rationale. Nat Rev Gastroenterol Hepatol. 2014;11:675-682.

22. Sindet-Pedersen S. Haemostasis in oral surgery-the possible pathogenetic implications of oral fibrinolysis on bleeding.
Experimental and clinical studies of the haemostatic balance in the oral cavity, with particular reference to patients with acquired and congenital defects of the coagulation system. Dan Med Bull. 1991;38:427-443.

23. Douglas LR, Douglass JB, Sieck JO, Smith PJ. Oral management of the patient with end-stage liver disease and the liver transplant patient. Oral Surg Oral Med Oral Pathol Oral Radiol Endod. 1998;86:55-64.

\section{Reprint requests:}

Jaana Helenius-Hietala, DDS, PhD

Department of Oral and Maxillofacial Diseases

University of Helsinki and Helsinki University Hospital

P.O. Box 63

FI-00014 Helsinki

Finland

Jaana.s.helenius@helsinki.fi 